\title{
Rapid Progression of Angioimmunoblastic T Cell Lymphoma Following BNT162b2 mRNA Vaccine Booster Shot: A Case Report
}

\author{
Serge Goldman ${ }^{1}$, Dominique Bron ${ }^{2}$, Thomas Tousseyn ${ }^{3}$, Irina Vierasu ${ }^{1}$, \\ Laurent Dewispelaere ${ }^{4}$, Pierre Heimann ${ }^{4}$, Elie Cogan ${ }^{5}$ and Michel Goldman ${ }^{6 *}$ \\ ${ }^{1}$ Department of Nuclear Medicine, Erasme Hospital, Université Libre de Bruxelles, Brussels, Belgium, ${ }^{2}$ Department of \\ Hematology, Jules Bordet Institute, Université Libre de Bruxelles, Brussels, Belgium, ${ }^{3}$ Department of Pathology, UZ Leuven \\ Hospitals, Leuven, Belgium, ${ }^{4}$ Laboratory of Hematology, LHUB, Université Libre de Bruxelles, Brussels, Belgium, \\ ${ }^{5}$ Department of Internal Medicine, CHIREC Hospital, Brussels, Belgium, ${ }^{6} / 3 \mathrm{~h}$ Institute, Université Libre de Bruxelles, \\ Brussels, Belgium
}

OPEN ACCESS

Edited by:

Ihsan Ullah,

Khyber Medical University, Pakistan

Reviewed by:

Tzvi Dwolatzky

Technion Israel Institute of

Technology, Israel

Laurent Pierre Nicod,

University of Lausanne, Switzerland

*Correspondence:

Michel Goldman

mgoldman@i3health.eu

Specialty section:

This article was submitted to

Pathology,

a section of the journal

Frontiers in Medicine

Received: 19 October 2021 Accepted: 08 November 2021 Published: 25 November 2021

Citation:

Goldman S, Bron D, Tousseyn T, Vierasu I, Dewispelaere L, Heimann P, Cogan E and Goldman M (2021)

Rapid Progression of Angioimmunoblastic T Cell Lymphoma Following BNT162b2 mRNA Vaccine

Booster Shot: A Case Report.

Front. Med. 8:798095.

doi: 10.3389/fmed.2021.798095
Since nucleoside-modified mRNA vaccines strongly activate $T$ follicular helper cells, it is important to explore the possible impact of approved SARS-CoV-2 mRNA vaccines on neoplasms affecting this cell type. Herein, we report and discuss unexpected rapid progression of lymphomatous lesions after administration of a BNT162b2 mRNA vaccine booster in a man recently diagnosed with AITL.

Keywords: mRNA vaccine, T cell, lymphoma, CovID-19, angioimmunoblastic, follicular

\section{INTRODUCTION}

The remarkable efficiency of nucleoside-modified SARS-CoV-2 mRNA vaccines has been related to their ability to induce a potent stimulation of $\mathrm{T}$ follicular helper (TFH) cells, resulting in persistent germinal center B cell responses $(1,2)$. Clinically, this might translate into reactive lymphoadenopathy which sometimes may raise a differential diagnosis with a lymphoproliferative disorder $(3,4)$. At the same time, the possible impact of SARS-CoV-2 mRNA vaccination on pre-existing peripheral $\mathrm{T}$ cell lymphoma is still to be determined.

\section{CASE REPORT}

A 66-year-old man with no significant medical history except for hypertension, hypercholesterolemia and type 2 diabetes presented on September 1, 2021 with cervical lymphadenopathies that became recently apparent during a flu-like syndrome. The two doses of BNT162b2 mRNA vaccine had been administered, respectively, 5 and 6 months earlier in the left deltoid. Besides moderate asthenia, he did not report any constitutional symptom. Blood examination indicated a mild inflammatory syndrome, without anemia or white blood cell changes; Lymphocytes immunophenotyping was unremarkable. Protein electrophoresis and immunoglobulin levels were normal and Coombs test was negative.

A 18F-FDG PET/CT revealed multiple voluminous hypermetabolic lymphadenopathies above and below the diaphragm as well as several extra-nodal hypermetabolic lesions (Figure 1, left panel). Considering a presumptive diagnosis of stage IV lymphoma, a left cervical lymph node biopsy was performed. Pathological examination revealed residual atrophic germinal centers, 


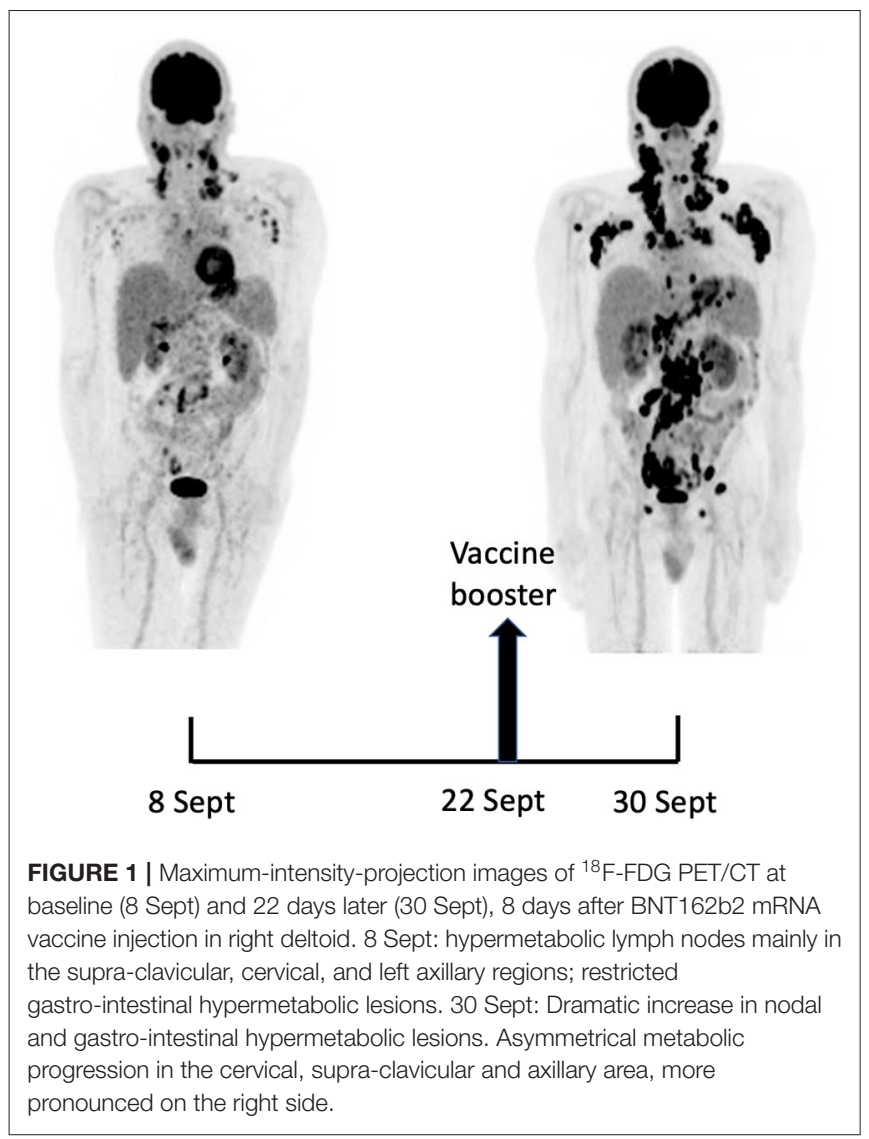

surrounded by an expanded paracortical area composed of an atypical T-cell infiltrate with clear cell morphology, expressing TFH cell markers (CD3, CD4, PD1, ICOS, BCL6, CXCL13) and a loss of CD7. The paracortical area contained an increased number of high-endothelial venules, supported by an increased number of follicular dendritic cell networks, with some foci of $\mathrm{EBV}+\mathrm{B}$-cell immunoblastic proliferation in the background (Figure 2). These features highly suggested a diagnosis of AngioImmunoblastic T cell Lymphoma (AITL), pattern 2. Next generation sequencing (NGS) performed on the biopsy specimen identified the RHOA G17V mutation characteristic of AITL (5) together with the DNMT3A, IDH2 and TET2 mutations. A TCR-gamma gene rearrangement confirmed a clonal $\mathrm{T}$ cell proliferation. Altogether, these findings unambiguously established the diagnosis of AITL. A bone marrow biopsy did not reveal neither morphological nor phenotypic abnormalities, but NGS revealed DMNT3A and TET2 mutations in bone marrow cells with allele frequencies of $41 \%$ and $36 \%$, respectively.

Fourteen days after the PET/CT, a booster dose of the BNT162b2 mRNA vaccine was administered in the right deltoid in preparation of the first cycle of chemotherapy. Within a few days following the vaccine booster, the patient reported noticeable swelling of right cervical lymph nodes. In order to get a baseline close to the initiation of the therapy, a second $18 \mathrm{~F}$ FDG PET/CT was performed 8 days after the vaccine booster administration, i.e. 22 days after the first one.
It demonstrated a clear increase in number, size and metabolic activity of pre-existing lymphadenopathies at the supra- and sub-diaphragmatic level. Furthermore, new hypermetabolic lymphadenopathies and new hypermetabolic sites had developed since the first examination, in several different locations (Figure 1, right panel). Total lesion glycolysis (TLG) index was used to assess the changes in lymph node activities (6). As compared with the initial test, there was a marked 5.3-fold increase in whole-body TLG, with the increase in the postbooster test being twice higher in the right axillary region than in the left one. In parallel, a mild increase in blood levels of ferritin, C-reactive protein and LDH were noted.

Methylprednisolone administration was initiated immediately after the 2nd PET/CT, followed by a first course of brentuximab vendotin combined with cyclophosphamide, doxorubicin (BVCHP) according to a recently published protocol (7). At the time of this report, 2 weeks after start of the treatment, clinical examination indicates significant decreased swelling of cervical and axillary lymph nodes, and the overall performance status of the patient is improving. Importantly, comparison of antiSARS-CoV-2 antibody levels immediately before and 21 days after the vaccine booster did not show a significant change in the production of anti-spike antibodies (171 vs.147 binding antibody units/ml).

\section{DISCUSSION}

Soon after the initiation of the anti-SARS-CoV-2 vaccination campaigns, it appeared that the injection of mRNA vaccines may induce swelling of lymph nodes draining the injection site. Although considered as benign, this vaccine reaction sometimes complicated the interpretation of 18F-FDG PET/CT imaging for suspicion of a neoplastic process affecting lymph nodes (3). When a lymph node biopsy was performed to exclude a malignant process, the pathological picture showed reactive benign changes with prominent germinal centers (3, 8). The differential diagnosis with lymphoma was occasionally complicated by the development of hypermetabolic sites at distance of the injection site, including contralateral lymph nodes or spleen $(9,10)$. In a patient with mantle lymphoma, PET/CT was suggestive of a relapse but was eventually excluded (11).

Published studies on hypermetabolic lymphadenopathy after SARS-CoV-2 vaccination were recently reviewed and the subject of a meta-analysis $(8,12)$. Most observations were reported after injection of approved nucleoside-modified mRNA vaccines, namely BNT162b2 (Pfizer-BioNTech) or mRNA-173 (Moderna) (8). Nevertheless, hypermetabolic lymphadenopathies were also observed in 31 health workers following injection of the adenovirus-vectored Vaxveria vaccine (13).

Considering oncologic patients, the most informative study was conducted in a series of 728 patients having received the BNT162b2 mRNA vaccine (14). PET/CT revealed hypermetabolic lymph nodes in the axillary and supraclavicular regions draining the vaccine injection site in $36 \%$ of the subjects having received the first dose and $54 \%$ of those studied after the 2 nd dose. The hypermetabolic lymph nodes were enlarged 


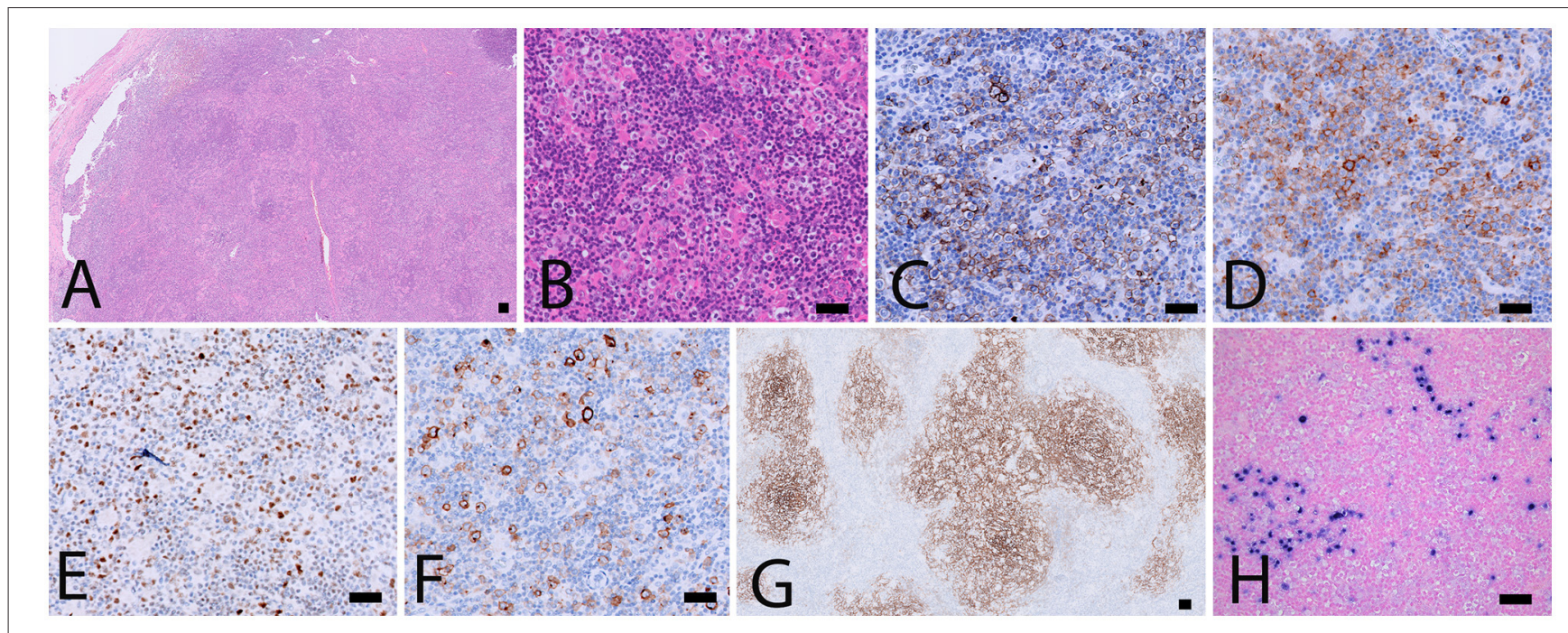

FIGURE 2 | Biopsy specimen. (A,B) H\&E stainings showing architectural disturbance due to a medium-sized lymphoid population with a clear cell morphology. (C-F) Immunohistochemical stainings establishing the TFH origin of the abnormal cell population: CD3+, CD4+, CD10+ (not shown), ICOS+ (C), PD1 (D), BCL6 (E) and expression of CD30 (F). CD21 staining (G) shows an extended network of follicular dendritic cells. (H) Intermediate sized EBV+ immunoblasts by EBER in situ hybridization. Scalebar: $100 \mu \mathrm{m}$.

in $7 \%$ of 1 st dose vaccinees and $18 \%$ of 2 nd dose vaccinees. Both differences were statistically significant, demonstrating that the impact on draining lymph nodes was greater after the booster dose, confirming data from the meta-analysis above (12). Regarding the relationship with the underlying malignancy, hypermetabolic lymph nodes were considered as malignant in $5 \%$ of the patients while no conclusion regarding the malignant nature could be drawn in $15 \%$ of the vaccinees including 16 patients with lymphoma. Interestingly, in none of these studies, the possibility that the mRNA vaccines could have played a role in the development of malignant lymph nodes was considered. Indeed, the consensus so far is that the occurrence of hypermetabolic lymphadenopathies should not question the safety of mRNA vaccines, neither in healthy individuals nor in patients with neoplastic conditions (15).

To the best of our knowledge, this is the first observation suggesting that administration of a SARS-CoV-2 vaccine might induce AITL progression. Several arguments support this possibility. First, the dramatic speed and magnitude of the progression manifested on two 18F-FDG PET-CT performed 22 days apart. Such a rapid evolution would be highly unexpected in the natural course in the disease. Since mRNA vaccination is known to induce enlargement and hypermetabolic activity of draining lymph nodes, it is reasonable to postulate that it was the trigger of the changes observed. Indeed, the increase in size and metabolic activity was higher in axillary lymph nodes draining the site of vaccine injection as compared to their contralateral counterparts. However, pre-existing lymphomatous nodes were also clearly enhanced as compared to the first test. Moreover, new hypermetabolic lesions most likely of lymphomatous nature clearly appeared at distance of the injection site.

In fact, the supposed enhancing action of the vaccine on AITL neoplastic cells is fully consistent with previous observations identifying TFH cells within germinal centers as key targets of nucleoside-modified mRNA vaccines both in animals and in man $(1,2)$. Malignant TFH cells, the hallmark of AITH, might be especially sensitive to mRNA vaccines when they harbor the RHOA G17V mutation which was present in our case. Indeed, this mutation facilitates proliferation and activation of several signaling pathways in TFH cells (16). Furthermore, mice genetically engineered to reproduce the RHOA G17V and TET2 mutations-both were present in our case-develop lymphoma upon immunization with sheep red blood cells (16). This experimental observation is relevant to RNA vaccines as RNA of sheep red blood cells was shown to be responsible for their ability to stimulate TFH and induce germinal center reaction (17).

Our case first raises the question of the COVID-19 prevention strategy to be used in this patient which is currently poorly protected against COVID-19. On the short term, the only option is to recommend strict masking and social distancing, and to offer him anti-SARS-CoV-2 antibody therapy in case of highrisk contact (16). On the longer term, the use of mRNA vaccines should clearly be avoided while other types of vaccines might be considered.

At this time, extrapolation of the findings of this case to other patients with AITL or other peripheral $\mathrm{T}$ cell lymphoma involving TFH cells is premature. AITL patients are rare and their mutation profile is heterogeneous. Furthermore, their immune reactions might be affected by their treatment. It is therefore unlikely that existing pharmacovigilance systems will be efficient to identify extremely rare cases like ours. Prospective studies involving systematic PET/CT imaging after SARS-CoV-2 vaccination in AITL patients with specified mutation profiles might eventually be needed. Whatever the result of such studies, it should not affect the overall favorable benefit-risk ratio of these much-needed vaccines. 


\section{CONCLUSION}

This observation, which has been posted as a pre-print on the SSRN platform (18), suggests that vaccination with the BNT162b2 mRNA vaccine might induce rapid progression of AITL. Dedicated studies are needed to determine whether this case can be extrapolated to populations of patients with AITL or other peripheral T cell lymphoma involving TFH cells.

\section{Patient Perspective}

The patient is the corresponding author of this case report. He hopes that this report will incentivize investigations to clarify the possible impact of anti-SARS-CoV-2 mRNA vaccination on the course of AITL. He remains convinced that mRNA vaccines represent very efficient products with a favorable benefit-risk ratio.

\section{DATA AVAILABILITY STATEMENT}

The original contributions presented in the study are included in the article/supplementary material, further inquiries can be directed to the corresponding author/s.

\section{ETHICS STATEMENT}

Ethical approval was not provided for this study on human participants because the retrospective analysis of the PET/CT data has been approved by the Ethics Committee of the Hôpital

\section{REFERENCES}

1. Pardi N, Hogan MJ, Naradikian MS, Parkhouse K, Cain DW, Jones L, et al. Nucleoside-modified mRNA vaccines induce potent $\mathrm{T}$ follicular helper and germinal center B cell responses. J Exp Med. (2018) 215:1571. doi: 10.1084/jem.20171450

2. Turner JS, O'Halloran JA, Kalaidina E, Kim W, Schmitz AJ, Zhou JQ, et al. SARS-CoV-2 mRNA vaccines induce persistent human germinal centre responses. Nature. (2021) 596:109-13. doi: 10.1038/s41586-021-03 738-2

3. Özütemiz C, Krystosek LA, Church AL, Chauhan A, Ellermann JM, Domingo-Musibay E, et al. Lymphadenopathy in COVID19 vaccine recipients: diagnostic dilemma in oncologic patients. Radiology. (2021) 300:E296-300. doi: 10.1148/radiol.202121 0275

4. Cohen D, Krauthammer SH, Wolf I, Even-Sapir E. Hypermetabolic lymphadenopathy following administration of BNT162b2 mRNA Covid19 vaccine: incidence assessed by [18F]FDG PET-CT and relevance to study interpretation. Eur J Nucl Med Mol Imaging. (2021) 48:185463. doi: 10.1007/s00259-021-05314-2

5. Sakata-Yanagimoto M, Enami T, Yoshida K, Shiraishi Y, Ishii R, Miyake Y, et al. Somatic RHOA mutation in angioimmunoblastic T cell lymphoma. Nat Genet. (2014) 46:171-5. doi: 10.1038/ng.2872

6. Kitadate A, Narita K, Fukumoto K, Terao T, Tsushima T, Kobayashi H, et al. Baseline total lesion glycolysis combined with interim positron emission tomography-computed tomography is a robust predictor of outcome in patients with peripheral T-cell lymphoma. Cancer Med. (2020) 9:550918. doi: $10.1002 / \mathrm{cam} 4.3226$

7. Horwitz S, O'Connor OA, Pro T B, Illidge, Fanale M, Advani R, et al. Brentuximab vedotin with chemotherapy for CD30-positive peripheral T-cell lymphoma (ECHELON-2): a global, double-blind, randomised, phase 3 trial. Lancet. (2019) 393:229-40. doi: 10.1016/S0140-6736(18)32984-2
Erasme (ref. P2017/020). Being one of the authors, the patient consented to the publication. The patients/participants provided their written informed consent to participate in this study. Written informed consent was obtained from the individual(s) for the publication of any potentially identifiable images or data included in this article.

\section{AUTHOR CONTRIBUTIONS}

All authors listed have made a substantial, direct, and intellectual contribution to the work and approved it for publication.

\section{FUNDING}

The department of nuclear medicine of Hôpital Erasme is financially supported by the Fonds Erasme and the Association Vinçotte Nuclear (Belgium).

\section{ACKNOWLEDGMENTS}

This paper was posted as a pre-print on the SSRN platform https://ssrn.com/abstract=3945001. We thank Alexandre De Wind and Denis Larsimont (Department of Pathology, Jules Bordet Institute, Brussels, Belgium), and Laurence de Leval (Institut Universitaire de Pathologie, CHUV, Lausanne, Switzerland) for additional analyses of the biopsy specimens, and Olivier Hermine (Department of Pathology, Necker Hospital, Paris, France) for useful discussion.

8. Keshavarz P, Yazdanpanah F, Rafiee F, Mizandari M. Lymphadenopathy following COVID-19 vaccination: imaging findings review. Acad Radiol. (2021) 28:1058-71. doi: 10.1016/j.acra.2021.04.007

9. Weeks J, O'Brien S, Rosenspire K, Dubroff J, Pantel A. evolving bilateral hypermetabolic axillary lymphadenopathy on FDG PET/CT following 2-dose COVID-19 vaccination. Clin Nucl Med. (2021) 46:10112 doi: 10.1097/RLU.0000000000003711

10. Nawwar AA, Searle J, Lyburn ID. Features of systemic immune response from COVID-19 vaccination on 18F-FDG PET/CT. Clin Nucl Med. (2021). doi: 10.1097/RLU.0000000000003859. [Epub ahead of print].

11. Xu G, Lu Y. COVID-19 mRNA vaccination-induced lymphadenopathy mimics lymphoma progression on FDG PET/CT. Clin Nucl Med. (2021) 46:353-4. doi: 10.1097/RLU.0000000000003597

12. Treglia G, Cuzzocrea M, Giovanella L, Elzi L, Muoio B. Prevalence and significance of hypermetabolic lymph nodes detected by 2-[(18)F]FDG PET/CT after COVID-19 vaccination: a systematic review and a metaanalysis. Pharmaceuticals. (2021) 14:762. doi: 10.3390/ph14080762

13. Shin M, Hyun CY, Choi YH, Choi JY, Lee K-H, Cho YS. COVID19 vaccination-associated lymphadenopathy on FDG PET/CT: distinctive features in adenovirus-vectored vaccine. Clin Nucl Med. (2021) 46:8149. doi: 10.1097/RLU.0000000000003800

14. Treglia G, Cuzzocrea M, Muoio B, Elzi L. PET findings after COVID-19 vaccination: "Keep Calm and Carry On." Clin Transl Imaging. (2021) 9:20914. doi: 10.1007/s40336-021-00430-3

15. Cortes JR, Ambesi-Impiombato A, Couronné L, Bernard OA, Ferrando AA. RHOA G17V induces $\mathrm{T}$ follicular helper cell specification and promotes lymphomagenesis. Cancer Cell. (2018) 33:259-73.e7. doi: 10.1016/j.ccell.2018.01.001

16. Loetsch C, Warren J, Laskowski A, Vazquez-Lombardi R, Jandl C, Langley DB, et al. Cytosolic recognition of RNA drives the immune response to heterologous erythrocytes. Cell Rep. (2017) 21:1624-38. doi: 10.1016/j.celrep.2017.10.044 
17. Cohen MS, Nirula A, Mulligan MJ, Novak RM, Marovich M, Yen C, et al. Effect of bamlanivimab vs placebo on incidence of COVID-19 among residents and staff of skilled nursing and assisted living facilities: a randomized clinical trial. JAMA. (2021) 326:46-55. doi: 10.1001/jama. 2021.8828

18. Goldman S, Bron D, Tousseyn T, Vierasu I, Dewispelaere L, Heimann P, et al. Rapid Progression of Angioimmunoblastic T Cell Lymphoma Following BNT162b2 mRNA Vaccine Booster Shot. A Case Report. Available online at: https://ssrn.com/abstract=3945001 (accessed October 18, 2021).

Conflict of Interest: The authors declare that the research was conducted in the absence of any commercial or financial relationships that could be construed as a potential conflict of interest.
Publisher's Note: All claims expressed in this article are solely those of the authors and do not necessarily represent those of their affiliated organizations, or those of the publisher, the editors and the reviewers. Any product that may be evaluated in this article, or claim that may be made by its manufacturer, is not guaranteed or endorsed by the publisher.

Copyright (๑) 2021 Goldman, Bron, Tousseyn, Vierasu, Dewispelaere, Heimann, Cogan and Goldman. This is an open-access article distributed under the terms of the Creative Commons Attribution License (CC BY). The use, distribution or reproduction in other forums is permitted, provided the original author(s) and the copyright owner(s) are credited and that the original publication in this journal is cited, in accordance with accepted academic practice. No use, distribution or reproduction is permitted which does not comply with these terms. 